\title{
UNIDAD DIDÁCTICA PARA LA ENSEÑANZA DE PROBABILIDAD MEDIADA POR UN OVA, ORIENTADA A UN COLEGIO RURAL DEL MUNICIPIO DE PAIPA
}

\section{DIDACTIC UNIT FOR THE PROBABILITY EDUCATION MEDIATED BY AN OVA, AIMED AT A RURAL SCHOOL IN THE MUNICIPALITY OF PAIPA}

\author{
Lic. Ruby Yamile Salcedo Ramírez *, PhD. Flavio Humberto Fernández Morales ** \\ PhD. Julio Enrique Duarte** \\ * Universidad Pedagógica y Tecnológica de Colombia, Instituto Santa Rita, Paipa. \\ E-mail: yamilesalcedo52@yahoo.com, ruby.salcedo@uptc.edu.co. \\ ** Universidad Pedagógica y Tecnológica de Colombia, Duitama, Colombia. \\ E-mail: flaviofm1@gmail.com, julioenriqued1@gmail.com
}

\begin{abstract}
Resumen: El presente trabajo muestra los resultados de aplicar una unidad didáctica que involucra la implementación de un Objeto Virtual de Aprendizaje, OVA, con el fin de mejorar las competencias en probabilidad, propuestas en los Estándares Básicos de Competencias en Matemáticas del Ministerio de Educación Nacional para el grado once. La investigación se enmarca dentro del enfoque cuasi experimental, ya que se busca la construcción de aprendizajes significativos en probabilidad, empleando para ello un diseño didáctico aplicado a un único grupo.
\end{abstract}

Palabras clave: Probabilidad, enseñanza de probabilidad, educación rural, OVA.

\begin{abstract}
The present work shows the results of applying a didactic unit that involves the implementation of a virtual object of learning, OVA, in order to improve the competitions in probability, proposals in the basic competitions standards in mathematics of the Ministry of National Education for the eleventh grade. The research is part of the quasiexperimental approach, since one looks for the construction of significant learnings in probability, using for this purpose a didactic design applied to an only group
\end{abstract}

Keywords: Probability competencies, probability teaching, rural education, OVA.

\section{INTRODUCCIÓN}

El desarrollo de competencias estadísticas y probabilísticas aportan a la construcción de herramientas por parte del estudiante, que lo ayudan a mejorar el razonamiento, toma de decisiones y deducción de situaciones que suceden a su alrededor (Riscanevo, 2016; Gómez y Rodríguez, 2014). Se desarrollan capacidades de recopilación, organización, representación e interpretación de datos e información, junto con la capacidad de identificar, describir y modelar situaciones del azar; así será posible estimar probabilidades de ocurrencia, dar predicciones y tomar decisiones, elementos fundamentales para los ciudadanos de hoy (Piratoba y Rojas, 2014; Burbano et al., 2017).

La enseñanza de temáticas relacionadas con ciencias básicas y aplicadas, como: física, química, electricidad, electrónica y mecánica, por mencionar algunas, se ha visto influida por las Tecnologías de la Información y la Comunicación, TIC (Angarita et al., 2014; Parra et al., 2014; Parada y Suárez, 2014; Guzmán-Luna Jaime et al., 2014). Las matemáticas, en especial la probabilidad y la 
estadística, también han sido objeto de la implementación de nuevas tecnologías para su enseñanza y aprendizaje (Valdés, 2011; García, 2013; Belfiori, 2014). Cada vez con mayor frecuencia, se encuentran propuestas que incluyen materiales educativos computarizados, objetos virtuales de aprendizaje, software especializado para enseñanza de conceptos matemáticos, todo ello complementado con el uso de plataformas para la administración de contenidos (Araujo y Romero, 2012; Torres y Duarte, 2016; González et al., 2016).

El Ministerio de Educación Nacional, MEN, ha propuesto una serie de competencias orientadas al mejoramiento del estudio de la probabilidad, las cuales se encuentran integradas en los estándares básicos de competencias en matemáticas (MEN, 2006). Sin embargo, la enseñanza de la probabilidad en grado once del Colegio Santa Rita, institución rural del municipio de Paipa, se orienta con base en el plan de área de matemáticas, el cual a su vez se retoma de un texto guía (Ardila et al., 2016).

Este texto trata con poca profundidad la temática de probabilidad, impidiendo que los estudiantes alcancen las competencias esperadas. Lo anterior, unido a un modelo educativo tradicional y con poca integración de las TIC, justifica la necesidad de diseñar una unidad didáctica enfocada al aprendizaje de la probabilidad, buscando la interacción con las nuevas tecnologías. Adicionalmente, con el servicio deficiente de internet en la institución, resulta práctica y adecuada la aplicación de Objetos Virtuales de Aprendizaje que puedan emplearse de forma local.

Este trabajo muestra los resultados obtenidos de una prueba diagnóstica aplicada a un grupo de estudiantes de grado once, pertenecientes al Colegio mencionado anteriormente, con el fin de determinar las competencias que poseen sobre la temática de probabilidad. Esta información se usó para estructurar un OVA, cuya mediación mejoró las competencias de los estudiantes, según se evidenció en la prueba final.

\section{MARCO TEÓRICO Y MÉTODOS}

\subsection{Objeto virtual de aprendizaje}

Los Objetos de Aprendizaje, OA, de acuerdo al portal Colombia Aprende, se definen como: "Un conjunto de recursos digitales, que pueden ser utilizados en diversos contextos, con un propósito educativo $y$ constituido por al menos tres componentes internos: contenidos, actividades de aprendizaje y elementos de contextualización" (MEN, s.f).

Sánchez (2014), indica que "Los OA son elementos de un nuevo tipo de instrucción basada en computadores, enmarcada en el paradigma orientado a objetos de las ciencias de la computación; la orientación a objetos valora altamente la creación de componentes (llamados objetos) que pueden ser reutilizados en múltiples contextos". La ventaja de los OA, a diferencia de los recursos tradicionales, es que cualquier persona puede acceder a ellos y emplearlos simultáneamente con otros (Jurado, 2010).

En otras palabras, los objetos de aprendizaje se pueden entender como cualquier objeto que incluye los entornos, estrategias, métodos, problemas o actividades, que se desarrollan de diversas formas y metodologías; lo anterior, en conjunto de los recursos digitales e informáticos (soportado por la tecnología) que se utilizan como apoyo en los procesos de enseñanza y aprendizaje (Valencia et al., 2014).

\subsection{Competencias en probabilidad}

En los Estándares Básicos de Competencias en Matemáticas se describen cinco tipos de pensamientos matemáticos: numérico, espacial, variacional, aleatorio y métrico (García et al., 2017). La probabilidad es parte del pensamiento aleatorio o probabilístico y los sistemas de datos, debido a la inmediata relación con la forma de predecir y tomar decisiones con respecto a la causalidad y el azar (Burbano et al., 2017).

Estos estándares se dividen en cinco grupos, en los cuales se presentan competencias subdivididas en los cinco pensamientos. A continuación se indican las competencias de los grados décimo y once, para el pensamiento aleatorio y sistemas de datos:

Interpreto y comparo resultados de estudios con información estadística provenientes de medios de comunicación.

Justifico o refuto inferencias basadas en razonamientos estadísticos a partir de resultados de estudios publicados en los medios o diseñados en el ámbito escolar. 
Diseño experimentos aleatorios (de las ciencias físicas, naturales o sociales) para estudiar un problema o pregunta.

Describo tendencias que se observan en conjuntos de variables relacionadas.

Interpreto nociones básicas relacionadas con el manejo de información como población, muestra, variable aleatoria, distribución de frecuencias, parámetros y estadígrafos).

Uso comprensivamente algunas medidas de centralización, localización, dispersión y correlación (percentiles, cuartiles, centralidad, distancia, rango, varianza, covarianza y normalidad).

Interpreto conceptos de probabilidad condicional e independencia de eventos.

Resuelvo y planteo problemas usando conceptos básicos de conteo y probabilidad (combinaciones, permutaciones, espacio muestral, muestreo aleatorio, muestreo con reemplazo).

Propongo inferencias a partir del estudio de muestras probabilísticas.

\subsection{Aprendizaje significativo}

El aprendizaje significativo es visto como aquel proceso en el que se relaciona la información ya adquirida con la nueva, construyendo nuevas ideas $\mathrm{y}$ conocimientos, que son aprendidos de forma clara y significativa, y pueden ser tomados como base para nuevas ideas estableciendo conexiones precisas entre estos conceptos y el ambiente que los rodea.

Como lo indica Díaz (2003), mencionando a Ausubel (1976), en el aprendizaje significativo el aprendiz relaciona la nueva información con los conocimientos que ya posee y con las experiencias previas. Por esto es fundamental la disposición de aprender que demuestre el aprendiz, así como la guía del docente y los materiales de apoyo, pues ellos fomentan y facilitan el aprendizaje significativo. El autor plantea que en este aprendizaje "se da sentido a lo aprendido, y se entiende su ámbito de aplicación y su relevancia en situaciones académicas y cotidianas" (p. 109).

Rodríguez (2004), menciona dos condiciones fundamentales para que se produzca aprendizaje significativo, a saber: predisposición para aprender de manera significativa y presentación de un material potencialmente significativo. El autor además menciona algunos aportes dados por Ausubel (2002), en cuanto a adquisición y retención del conocimiento; una perspectiva cognitiva en donde indica que: “... aprender significativamente o no forma parte del ámbito de decisión del individuo, una vez que se cuenta con los subsumidores relevantes y con un material que reúne los requisitos pertinentes de significatividad lógica” (Pág. 4).

De acuerdo con lo anterior, el aprendizaje puede llegar a ser cada vez más significativo si los estudiantes son capaces de generar diversos modelos que los lleven a explicar y predecir, con mayor certeza, algunas de las situaciones que se les presenten en su entorno, viéndolo como la comprensión, y capacidad de trasferencia, que genera más ideas relacionadas con una imagen o símbolo ya significativo.

\subsection{Unidad didáctica}

La definición más pertinente para Unidad Didáctica, UD, es la que presenta Corrales (2010) citando a Escamilla (1992):

"La UD es una forma de planificar el proceso de enseñanza-aprendizaje alrededor de un elemento de contenido que se convierte en eje integrador del proceso, aportándole consistencia y significatividad." De esta forma se ven integrados aquellos conocimientos con las experiencias contextualizadas dentro del proceso para así adecuarlo al estudiante y perfeccionarlo a medida que se trabaja.

Es fácil comprender por qué la unidad didáctica es considerada como un instrumento en el que se presentan un conjunto de actividades elaboradas con ciertos parámetros curriculares (objetivos, contenidos, actividades y evaluación) de forma que logre una relación y un aprendizaje significativo entre los actores que se involucran. Como lo menciona Rodríguez (2008), el diseño de la unidad didáctica debe realizarse por medio de tres pasos articulados de tal forma que sea posible el descubrimiento de los ejes y contenidos fundamentales, y así recalcarlos para "proceder con la secuenciación según los principios de la organización psicológica del conocimiento" (p. 7)

La estructura de la Unidad Didáctica propuesta, UD, busca un aprendizaje significativo de la probabilidad y contiene los objetivos de aprendizaje, contenidos, metodología empleada, 
recursos humanos y materiales, tiempo $\mathrm{y}$ evaluación. Los contenidos de la UD se organizaron en 5 unidades, que integran contenidos específicos del tema a tratar, a saber:

Unidad 1: Conceptos básicos: Espacio muestral y experimentos aleatorios

Unidad 2: Sucesos aleatorios, clases y operaciones entre sucesos

Unidad 3: Técnicas de conteo

Unidad 4: Regla de Laplace

Unidad 5: Propiedades de probabilidad

En las sesiones de clase previstas para las unidades temáticas, se iniciará con la introducción al tema y su contextualización; luego se brindará la teoría necesaria de acuerdo al texto guía de la institución; a partir de ejemplos y ejercicios desarrollados durante la clase, se aclararán las dudas o ideas erróneas sobre los conceptos tratados. Finalmente, se efectuará la valoración del aprendizaje realizado.

\subsection{Metodología}

La metodología de esta investigación implica un estudio cuasi experimental, ya que lo que se busca con el desarrollo de este proyecto es la construcción de aprendizajes significativos, mediados por un OVA, en una temática relacionada con probabilidad, tratada en un colegio de zona rural. Como lo indica Cabré (2012), los diseños cuasi-experimentales no son aleatorios, además los requisitos no son tan estrictos como en la investigación experimental; por tanto, los datos no pueden ajustarse y no son del completo control de los investigadores.

La unidad didáctica se implementó en el Colegio Fundación Santa Rita, ubicado en el corregimiento del Pantano de Vargas, municipio de Paipa, Boyacá; gran parte de los estudiantes son de esta zona, pertenecientes a los estratos 1 y 2 , con un núcleo familiar incompleto; la comunidad de esta zona se caracteriza por el desarrollo de actividades agrícolas y ganaderas. La mayoría de los padres de familia presentan bajo grado de escolaridad, no culminaron la primaria ya que se dedicaron a la labor agrícola y/o al cuidado de los hijos.

La población objeto de estudio está conformada por 9 estudiantes de núcleo común, pertenecientes a grado once de la institución, con edad promedio de 16 años ( 5 mujeres y 4 hombres), de los cuales 6 estudiantes no tienen acceso a computador en sus casas; la zona rural en la que se ubica el colegio dificulta el acceso a internet y los equipos de la institución son obsoletos ante el avance tecnológico actual. Por ello, se hizo uso de los dispositivos de que disponen los mismos estudiantes (3 computadores portátiles) y el computador portátil de 3 docentes de la institución.

La intensidad horaria para el área de matemáticas en todos los grados es de 6 horas semanales, cada una de ellas equivalente a 50 minutos. En el grado once se distribuyen las clases de acuerdo a las asignaturas de cálculo (4 horas), geometría (1 hora) y estadística/probabilidad (1 hora). El tiempo de trabajo en el aula para la implementación de la unidad didáctica fue de 2 meses, dentro del espacio asignado para estadística/probabilidad en el horario de clases.

Las siguientes fueron las actividades desarrolladas para cumplir el objetivo propuesto:

- Prueba diagnóstica para establecer el estado inicial y los preconceptos que los estudiantes poseen acerca de la temática de probabilidad.

- Identificación de los requerimientos técnicos y pedagógicos para el OVA, con el fin de que se adapte a las características de la población.

- Desarrollo de la unidad didáctica propiamente dicha, incluyendo la elaboración del OVA como parte integral del ambiente de aprendizaje.

- Validar la unidad didáctica con los estudiantes, lo cual permite establecer la funcionalidad del OVA dentro del proceso formativo.

\section{RESULTADOS Y DISCUSIÓN}

\subsection{Prueba diagnóstica}

Con el fin de establecer el estado inicial de los estudiantes frente a los conceptos básicos en probabilidad, se planteó un cuestionario con siete situaciones problema enfocadas a la valoración de las competencias básicas en probabilidad que propone el MEN; en la tabla 1 se relacionan tres de estas competencias con la situación planteada dentro de la prueba.

Las respuestas de la prueba diagnóstica fueron valoradas a partir del planteamiento, procedimiento desarrollado y respuesta obtenida en cada una de las situaciones formuladas en el cuestionario; los resultados se clasificaron en correctos, incorrectos y sin solución. La tabla 2 muestra las competencias a desarrollar y reforzar, junto con la cantidad de estudiantes clasificados de acuerdo a los resultados en cada una de las situaciones del cuestionario. 


\section{Tabla 1: Relación competencia y Situación problema en la prueba diagnóstica}

\begin{tabular}{|c|c|}
\hline Competencia & Situación planteada dentro de la prueba \\
\hline $\begin{array}{l}\text { Resuelvo y planteo } \\
\text { problemas usando } \\
\text { conceptos básicos. } \\
\text { Interpreto conceptos } \\
\text { de conteo y } \\
\text { probabilidad. }\end{array}$ & $\begin{array}{l}\text { 1. Una familia conformada por tres } \\
\text { personas: A, B y C, que hacen parte de } \\
\text { un grupo de estudio que siempre tiene } \\
\text { un orientador en cada uno de los } \\
\text { salones: } 1,2 \text { y } 3 \text {. Un día en específico, } \\
\text { a cada uno de los miembros de la } \\
\text { familia le corresponde un orientador al } \\
\text { azar. Si es requerido el registro del } \\
\text { número de salón asignado a cada } \\
\text { miembro de la familia, los posibles } \\
\text { resultados serían: }\end{array}$ \\
\hline
\end{tabular}

2. Una persona tiene para ir desde su casa al trabajo cuatro caminos diferentes, para ir del trabajo al restaurante otras cinco, mientras que de la casa al restaurante sólo son dos. Si la ruta varía según la hora y el medio de transporte que se utilice ¿de cuantas maneras puede hacer este recorrido?

3. En un juego, se extraen sucesivamente tres pimpones de una bolsa que contiene 6 blancas, 8 rojas y 10 verdes. ¿Cuál es la probabilidad de sacar, en orden, un pimpón rojo, blanco y verde?

4. En un colegio hay 200 estudiantes matriculados en bachillerato, de ellos

Justifico o refuto inferencias basadas en razonamientos estadísticos.

Interpreto conceptos de probabilidad condicional e independencia de eventos.
150 estudian inglés, 80 juegan baloncesto $\mathrm{y}, 40$ juegan baloncesto $\mathrm{y}$ estudian inglés. Plantee una tabla que organice los datos suministrados anteriormente. Calcule la probabilidad de que al seleccionar una persona esta sea: a. Un estudiante que juegue baloncesto y estudie inglés; b. Un estudiante que solo estudie inglés; c. Un estudiante que solo juegue baloncesto.

5. Un grupo de personas distribuidos de la siguiente forma: 27 mujeres y 23 hombres, además entre ellos hay 15 mujeres y 8 hombres que son zurdos. Organice los datos suministrados anteriormente. Calcule la probabilidad de que al seleccionar una persona esta sea: a. Un hombre zurdo; b. Sea una persona diestra; c. Una mujer diestra; d. Sea hombre
6. En un estudio realizado en un colegio para determinar la cantidad de celulares que tienen los 20 alumnos de grado once, de ellos 7 son hombres de los cuales 4 tienen celular y 8 de las mujeres también. Complete la siguiente tabla y responda: Calcule la probabilidad de que al seleccionar una persona esta: a. Sea un hombre sin celular; b. Un alumno con celular; c. Sea una mujer con celular; d. Sea mujer

Resuelvo y planteo problemas usando conceptos básicos.

7. Para la siguiente tabla, plantee una situación con una pregunta resuelta:

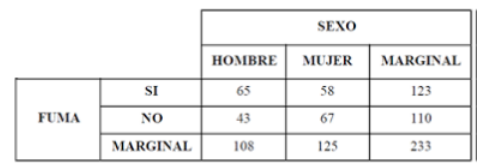

Tabla 2: Resultados prueba inicial

\begin{tabular}{|c|c|c|c|c|}
\hline \multirow{2}{*}{ Competencia } & \multirow{2}{*}{$\begin{array}{l}\text { Situación } \\
\text { en la } \\
\text { prueba }\end{array}$} & \multicolumn{3}{|c|}{$\begin{array}{l}\text { Resultados de los } \\
\text { estudiantes }\end{array}$} \\
\hline & & $\begin{array}{l}\text { Incor } \\
\text { recta }\end{array}$ & $\begin{array}{l}\text { Corre } \\
\text { cta }\end{array}$ & $\begin{array}{l}\text { Sin } \\
\text { Solución }\end{array}$ \\
\hline \multirow{3}{*}{$\begin{array}{l}\text { C1. Resuelvo y } \\
\text { planteo problemas } \\
\text { usando conceptos } \\
\text { básicos. Interpreto } \\
\text { conceptos } \\
\text { conteo } \\
\text { probabilidad. }\end{array}$} & 1 & 0 & 9 & 0 \\
\hline & 2 & 7 & 0 & 2 \\
\hline & 3 & 5 & 0 & 4 \\
\hline \multirow{3}{*}{$\begin{array}{lr}\text { C2. Justifico } & \text { o } \\
\text { refuto inferencias } \\
\text { basadas } & \text { en } \\
\text { razonamientos } & \\
\text { estadísticos. } & \\
\text { Interpreto } & \\
\text { conceptos } & \text { de } \\
\text { probabilidad } & \\
\text { condicional } & \text { e } \\
\text { independencia } & \text { de } \\
\text { eventos. } & \end{array}$} & 4 & 9 & 0 & 0 \\
\hline & 5 & 9 & 0 & 0 \\
\hline & 6 & 9 & 0 & 0 \\
\hline $\begin{array}{l}\text { C3. Resuelvo y } \\
\text { planteo problemas } \\
\text { usando conceptos } \\
\text { básicos. }\end{array}$ & 7 & 4 & 4 & 1 \\
\hline
\end{tabular}

En la tabla 2 se observa que los estudiantes poseen falencias en las tres competencias valoradas por las situaciones de la tabla 1. En este sentido, para el planteamiento del espacio muestral (competencia $1, \mathrm{C} 1)$ necesitaron la enumeración de los datos que debían relacionar para la solución de problemas usando conceptos básicos. En lo que respecta a inferencias basadas en razonamientos estadísticos (competencia 2, C2), la confusión se relacionó con la probabilidad condicional pues los 9 estudiantes fallan en el análisis de los eventos cuando se plantean dos características a un solo elemento. Sin 
embargo, en el planteamiento de situaciones a partir de datos dados (competencia 3, C3), 4 de los 9 estudiantes lo hacen de forma coherente, mientras que los 5 restantes presentan falencias en la aclaración de la información que se pide mostrar, justificar y resolver; esto indica las dificultades de la mayoría de los estudiantes a la hora de plantear problemas usando conceptos básicos de conteo y probabilidad.

Estos resultados respaldan la necesidad de implementar estrategias que favorezcan el aprendizaje significativo en la temática de probabilidad, buscando con ello el mejoramiento de estas competencias dentro del área de matemáticas. Además, la prueba diagnóstica se constituye en un insumo fundamental para elaborar una unidad didáctica basada en un OVA, que complemente la enseñanza de la probabilidad en grado once, empleando para ello estrategias que motiven a los estudiantes y los involucre en la solución de problemas del contexto.

\subsection{El OVA propuesto}

El Objeto Virtual de Aprendizaje, OVA, se desarrolló a partir de la identificación de los requerimientos funcionales, entendidos como la descripción de aquellos servicios y funciones para su aplicación (Reyes et al., 2015). Entre ellos se tienen: la visualización del contenido abordado, la presentación de la temática en forma clara y ordenada, de modo que permita al estudiante comprender cada uno de los conceptos tratados; igualmente se consideran actividades para practicar y afianzar el conocimiento adquirido, realizando al mismo tiempo un proceso de retroalimentación y evaluación de los conceptos desarrollados; la gestión del contenido se hace por medio de imágenes, actividades y ejemplos de forma sencilla.

El contenido se estableció a partir de las deficiencias identificadas en la prueba diagnóstica, y se basa en las cinco unidades temáticas propuestas en la sección 2.4. El OVA se elaboró con el software Adobe Dreamweaver, de acuerdo con los componentes para un material de este tipo.

En su mayoría, los OVA presentan una estructura que busca contribuir al aprendizaje del estudiante; dentro de estos componentes se encuentran las palabras clave y los objetivos, que expresan explícitamente lo que se va a aprender. Luego están los contenidos y ejemplos en donde se da a conocer el tema, mediante la representación del conocimiento en definiciones, explicaciones y tareas a realizar, con el fin de hacer significativo el aprendizaje. Finalmente están la evaluación y retroalimentación, para corroborar el correcto conocimiento del tema y buscando el mejoramiento de su aprendizaje.

Bajo esta estructura se presentan los contenidos básicos de probabilidad incluidos en el OVA, los cuales permiten desarrollar las competencias propuestas en la tabla 1. Estos contenidos se corresponden con los de la UD y con los presentados en el texto guía que sigue la institución. La estructura de cada tema toma como fundamento la explicación de las definiciones, ejemplos contextualizados, desarrollo de ejercicios planteados durante la clase y una valoración de los conocimientos adquiridos.

El OVA inicia con una portada como se muestra en la figura 1, la cual presenta el concepto de probabilidad junto con una imagen relacionada y las temáticas a reforzar. Cada uno de los temas se presenta con la misma estructura.

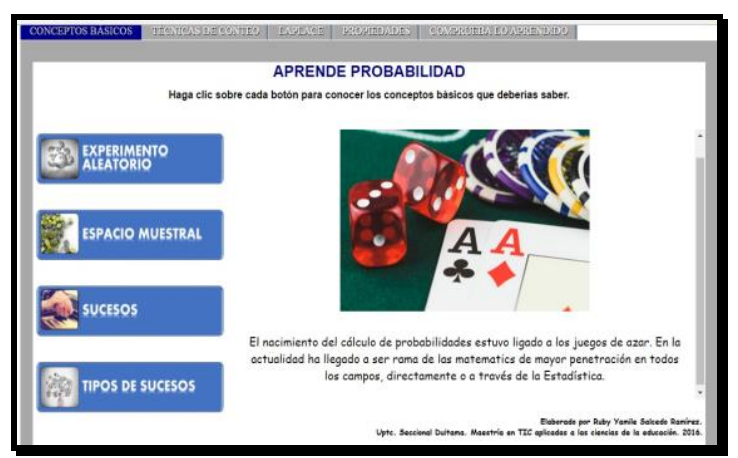

Fig. 1. Portada del OVA

\subsection{Estrategia didáctica}

En el modelo tradicional que se viene empleando en la institución se desarrollan las temáticas de forma magistral, siguiendo el proceso de "Título, definición, ejemplo, ejercicios y evaluación", guiados por un texto definido por la misma, proceso que ha impedido que los estudiantes alcancen las competencias establecidas por el MEN. En este sentido, se implementó la UD de la sección 2.4, enfocada al aprendizaje de la probabilidad, cuya implementación está mediada por el OVA descrito en la sección precedente.

La estrategia didáctica se adelantó en la institución dentro del tiempo destinado a la asignatura, durante dos meses, con sesiones de 50 minutos semanales cada una. En este periodo se desarrollaron clases 
con metodología tradicional, por ser esta la establecida por la institución. En las actividades se integró el OVA como recurso de retroalimentación y refuerzo de la temática, el cual se realiza a través de algunas situaciones problema planteadas para su aplicación.

Los participantes directos en esta experiencia expresaron sus opiniones sobre el OVA usado durante las clases, algunas positivas como por ejemplo:

“Me gustó mucho el programa que se utilizó en la clase por que no habíamos trabajado en matemáticas nada de eso, y porque en el colegio no nos dejan usar los computadores en otras clases, solo en informática, ojalá nos dejen seguir utilizándolos más”.

"Fue chévere trabajar las clases así, con programas diferentes y no solo copiando en el cuaderno, también estuvieron chéveres las actividades como en forma de juegos".

Entre otras, las siguientes sugerencias de los estudiantes pueden tomarse como punto de partida para mejorar el OVA:

"Estuvo chéveres las clases así, pero le hubiesen colocado videos para que fuera más emocionante, pero en general aprendi mejor".

"Faltaron colores más alegres, para que llamara la atención, para que no se desconcentrara en las clases".

La implementación del OVA y en general de la unidad didáctica, permitió evidenciar el cambio de actitud en los estudiantes, ya que no es común el desarrollo de las sesiones de clase del área con este tipo de ayudas tecnológicas. Entre las dificultades se pueden mencionar: las limitaciones frente al modelo pedagógico tradicional que sigue la institución, el poco tiempo destinado al desarrollo de las temáticas de probabilidad, la falta de instalaciones apropiadas para la integración de la tecnología en las clases, así como el limitado acceso a internet por la ubicación de la institución. Estos inconvenientes son reportados con frecuencia en la literatura en los diversos niveles educativos y no son exclusivos de las temáticas de probabilidad, por lo que deben destinarse esfuerzos en la formación inicial y continua de los docentes en el uso de estrategias didácticas innovadoras (Bernal, 2017; Peré, 2017; Núñez, 2015; Soto et al., 2014).

\subsection{Prueba final}

La prueba final consistió en un cuestionario de siete situaciones similares a las de la prueba diagnóstica, cuyo resultado se presenta en la tabla 3.

Tabla 3: Resultados prueba final

\begin{tabular}{lcccc}
\hline \multirow{2}{*}{$\begin{array}{l}\text { Compet } \\
\text { encia }\end{array}$} & $\begin{array}{l}\text { Situación } \\
\text { en la } \\
\text { prueba }\end{array}$ & \multicolumn{4}{c}{ Resultados de los estudiantes } \\
\cline { 2 - 5 } & 1 & 0 & 9 & 0 \\
\hline \multirow{2}{*}{ C1. } & 2 & 3 & 6 & 0 \\
\cline { 2 - 5 } & 3 & 2 & 7 & 0 \\
\hline & 4 & 4 & 4 & 1 \\
C2. & 5 & 2 & 7 & 0 \\
\hline & 6 & 3 & 6 & 0 \\
\hline C3. & 7 & 3 & 5 & 1 \\
\hline
\end{tabular}

En la tabla 3 se observa que los estudiantes presentan avances en el desarrollo de situaciones enfocadas a las tres competencias de la tabla 1. Los estudiantes mejoraron en el planteamiento de espacios muestrales y en el conteo de los posibles resultados en distintas situaciones, aunque pueden fallar en el proceso de organización de los datos, lo que dificulta el conteo. Además, los estudiantes mejoraron en el cálculo de probabilidades de algunas situaciones a partir del planteamiento de dos características en los datos; sin embargo, en el proceso de organización en diagramas de Venn, se siguen presentando falencias lo que lleva a determinaciones erróneas en el cálculo de probabilidades a partir de los datos.

La mayor dificultad está en el planteamiento de problemas debido al léxico limitado y la poca argumentación a la hora de expresar ideas de forma coherente y clara, de los 9 estudiantes 4 no desarrollaron la situación como se esperaba. La habilidad para interpretar el enunciado de las situaciones problema y su transformación al lenguaje matemático, es una barrera importante a la hora de mejorar la competencia de resolución de problemas por parte de los estudiantes (Hernández et al., 2017; Da Silveira, 2017).

Al comparar los resultados obtenidos en las pruebas diagnóstica y final, ver tablas 2 y 3 , se evidencia una mejora en las competencias en lo que respecta a la resolución, interpretación y análisis de problemas a partir de conceptos básicos y la determinación de probabilidades. 
Lo anterior permite afirmar que el uso de un OVA como apoyo a la unidad didáctica, facilitó el desarrollo de las competencias previstas. Esto se debe a que el OVA resultó atractivo y motivador para los estudiantes, facilitando el desarrollo de las actividades propuestas de forma dinámica; el OVA se convierte en un instrumento de retroalimentación y evaluación de los conocimientos adquiridos, pues su contenido brinda una síntesis de lo visto durante las clases.

\section{CONCLUSIONES}

El principal objetivo del proyecto fue desarrollar una unidad didáctica mediada por un Objeto Virtual de Aprendizaje, OVA, para mejorar tres de las competencias básicas de probabilidad establecidas para el grado once por el Ministerio de Educación Nacional, MEN. El lugar en el que se llevó a cabo la experiencia, un colegio rural de índole tradicional con deficiencias en infraestructura tecnológica y casi nulo servicio de internet, justifica la elección del OVA como mediador del aprendizaje, ya que este no requiere acceso a internet para su utilización.

El OVA se elaboró a partir de los resultados de la prueba diagnóstica, como una herramienta para lograr un aprendizaje significativo de la temática de probabilidad. Las actividades propuestas en el OVA tienen el propósito de fomentar en los estudiantes el análisis y planteamiento de soluciones a diversos problemas del contexto. Además, las actividades de evaluación y retroalimentación fortalecen el proceso de aprendizaje y fomentan el autoaprendizaje en los estudiantes.

Luego de utilizar el OVA en el desarrollo de la unidad didáctica, los resultados de la prueba final permitieron establecer que hubo una mejora en las competencias que se quería desarrollar, a saber: la resolución, interpretación y planteamiento de problemas usando conceptos básicos; la correcta aplicación de los principios de conteo y el cálculo de probabilidades.

La principal dificultad se presenta en el planteamiento de problemas debido al limitado léxico y argumentación de los estudiantes, a la hora de expresar ideas de forma coherente y clara. Este hecho indica la necesidad de desarrollar estrategias didácticas orientadas a fortalecer la competencia comunicativa, junto con la integración de situaciones problema que permitan su transformación al lenguaje matemático, promoviendo así la competencia de resolución de problemas por parte de los estudiantes.

\section{REFERENCIAS}

Angarita V., M. A., Fernández M., F. H., \& Duarte, J. E. (2014). La didáctica y su relación con el diseño de ambientes de aprendizaje: una mirada desde la enseñanza de la evolución de la tecnología. Revista de Investigación, Desarrollo e Innovación, Vol. 5, No. 1, 46-55. Doi: $10.19053 / 20278306.3138$

Araujo, D., y Romero, S. (2012). Uso de las TIC en el proceso enseñanza aprendizaje. Universidad de la Guajira Colombiana. TÉLÉMATIQUE, Vol. 11, No. 1, 69-83. Recuperado de: http://www.redalyc.org/ articulo.oa?id=78423414005

Ardila G., V. H., Dueñas Á., M. F., Bogotá T., M., Cañón G., M. A., Alfonso O., M. A., Perdomo P., A. C. y Abella P., L. E. (2016). Aplica: Matemáticas 11. Bogotá, Colombia: Ediciones SM.

Belfiori, L. (2014). Enseñanza de estadística con recursos TIC. Congreso Iberoamericano de Ciencia, Tecnología, Innovación y Educación. Buenos Aires (Argentina). Recuperado de http://scholar.googleusercontent.com/scholar? q=cache:ASIJgmESXB8J:scholar.google.com/ +Belfiori+(2014)\&hl=es\&as_sdt=0,5

Bernal P., M. L. (2017) ¿qué escriben los niños?, una mirada desde el modelo escuela nueva. Revista de Investigación, Desarrollo e Innovación, Vol. 7, No. 2., 255-268. Doi: 10.19053/20278306.v7.n2.2017.6069

Burbano P., V. M. A., Valdivieso M., M. A., y Aldana B., E. (2017) Conocimiento base para la enseñanza: un marco aplicable en la didáctica de la probabilidad. Revista de Investigación, Desarrollo e Innovación, Vol. 7, No. 2, 269-285. Doi: 10.19053/20278306.v7.n2.2017.6070

Cabré, R. B. (2012). Diseños cuasi-experimentales y longitudinales. 1 - 86. Recuperado de: http://diposit.ub.edu/dspace/bitstream/2445/30 783/1/D.\%20cuasi\%20y\%20longitudinales.pd $\mathrm{f}$

Corrales, A. (2010). La programación a medio plazo dentro del tercer nivel de concreción: Las unidades didácticas. Revista Digital de Educación Física, Año 1, No. 2. , 41-53. Recuperado de: http://emasf.webcindario.com/La_programaci 
on_a_medio_plazo_dentro_del_tercer_nive_\% 20de_concrecion_unidades_didacticas.pdf

Díaz B., F. (2003). Cognición situada y estrategias para el aprendizaje significativo. REDIE. Revista Electronica de Investigacion Educativa, Vol. 5, No. 2. Recuperado de http://www.redalyc.org/pdf/155/15550207.pdf

García G, R. (2013). Aprendizaje de la estadística y la probabilidad en secundaria. España. Recuperado de: http://repositorio.unican.es/xmlui/bitstream/ha ndle/10902/2939/Garcia\%20Garcia\%20Ricard o.pdf?sequence $=1$

Da Silveira, M. (2017). Compreensão da matemática no uso de símbolos e da gramática. Revista Guillermo de Ockham, Vol. 15, No. 1. Doi: http://dx.doi.org $/ 10.21500 / 22563202.3190$

García Q, B., Coronado, A., y Giraldo O., A. (2017). Implementación de un modelo teórico a Priori de competencia matemática asociado al aprendizaje de un objeto matemático. Revista de Investigación, Desarrollo e Innovación, Vol. 7. No. 2, 301-315. Doi: 10.19053/20278306.v7.n2.2017.60 72

Gómez V., N. Y., y Rodríguez G., J. K. (2014). Análisis de la producción científica matemática en Colombia. Base web of knowledge (2001-2012). BISTUA Revista de la Facultad de Ciencias Básicas, Vol. 12, No. 2, 70-84. doi: https://doi.org/10.24054/01204211.v2.n2.2014 .1660

González N., S., Fernández M., F., y Duarte, J. (2016). Memoria de trabajo y aprendizaje: Implicaciones para la educación. Saber, Ciencia y Libertad, Vol. 11, No. 2, 161-176. Doi: 10.22525/sabcliber.2016v11n2.16 1176

Guzmán-Luna Jaime, Ingrid-Durley Torres, Juan Felipe Alvarez (2014). Propuesta de un generador de aplicaciones educativas basadas en televisión digital usando arquitectura de cómputo en la nube. Revista Colombiana de Tecnologías de Avanzada ISSN: 1692-7257 Volumen 2 - Número 24.

Hernández S., C., Prada N., R., y Gamboa S., A. (2017). Conocimiento y uso del lenguaje matemático en la formación inicial de docentes en matemáticas. Revista de Investigación, Desarrollo e Innovación, Vol. 7, No. 2, 287-299. Doi: 10.19053/202783 06.v7.n2.2017.6071

Jurado E., G. E. (2010). Objetos virtuales de aprendizaje (OVA) como mediadores del proceso de aprendizaje. Revista Criterios, 63 $72 . \quad$ Recuperado de: http://www.umariana.edu.co/ojseditorial/index.php/criterios/article/download/ $283 / 245$

Ministerio de Educación Nacional, MEN. (2006). Estándares Básicos de Competencias en Matemáticas. Recuperado de: http://www.mineducacion.gov.co/1621/article s-116042_archivo_pdf2.pdf

Ministerio de Educación Nacional, MEN (s.f.). Portal Colombia aprende. Recuperado de: http://www.colombiaaprende.edu.co/html/dire ctivos/1598/propertyvalue-34418.html

Núñez P., V. (2015). Pedagogía social e interculturalismo: una lectura posible. Revista de Investigación, Desarrollo e Innovación, Vol. 5, No. 2, 141-149. Doi: 10.19053/20278306.3716

Parada H., A., y Suárez A., Z. E. (2014). Influencia de las Tecnologías de la Información y la Comunicación en la apropiación de conceptos de electrónica análoga, en estudiantes de grado séptimo de educación básica. Revista de Investigación, Desarrollo e Innovación, Vol. 5, No. 1, 20-31. Doi: 10.19053/20278306.3137

Parra L., L. F., Duarte, J. E., y Fernández M., F. H. (2014). Propuesta didáctica para la enseñanza de circuitos eléctricos básicos. Revista de Investigación, Desarrollo e Innovación, Vol. 4, No. 2, 138-147. Doi: $10.19053 / 20278306.2891$

Peré, N. (2017). Apuntes para analizar la relación entre innovación, TIC y formación pedagógico-didáctica. PRAXIS \& SABER, Vol. 8, No. 16, 15 - 33. Doi: 10.1905 3/22160159.v8.n16.2017.6165

Piratoba G., R. P., y Rojas M., C. E. (2014). Cambios en las concepciones iniciales e inducidas sobre la naturaleza de las matemáticas y su didáctica, en estudiantes de un programa de Licenciatura en Matemáticas y Estadística. Revista de Investigación, Desarrollo e Innovación, Vol. 5, No. 1, 32-45. Doi: 10.19053/20278306.3140

Reyes C., F., Fernández M., F. H., y Duarte, J. E. (2015). Herramienta para la selección de software educativo aplicable al área de tecnología en educación básica. ENTRAMADO, Vol. 11, No. 1, 186-193. Doi: 10.18041/entramado.2015v11n1.21101

Riscanevo E., L. (2016). La teoría de la práctica social del aprendizaje en la formación de 
profesores de matemáticas. Revista de Investigación, Desarrollo e Innovación, Vol. 7, No. 1, 93-110. Doi: 10.19053/20278306.v7.n1.2016.5635

Rodríguez, J. M. (2008). Algunas teorías para el diseño instructivo de unidades didácticas. Unidad didáctica: "El alfabeto griego". Recuperado http://www.um.es/ead/red/20/rodriguez.pdf

Rodríguez P., M. (2004). La teoría del aprendizaje significativo. Primera Conferencia Internacional sobre Mapas Conceptuales. Recuperado de: http://cmc.ihmc.us/papers/cmc2004-290.pdf

Sánchez M., I. (2014). Estado del arte de las metodologías y modelos de los objetos virtuales de aprendizaje (OVAs) en Colombia. ENTORNOS, 93-107. Recuperado de: http://journalusco.edu.co/index.php/magazine/ article/view/472

Soto J., J. C., Franco E., M. L., y Giraldo C., J. C. (2014). Desarrollo de una metodología para integrar las TIC (Tecnologías de la Información y la Comunicación) en las IE (Instituciones Educativas) de Montería. Zona Próxima, No. 21, 34-51. Recuperado de: http://www.scielo.org.co/scielo.php?script=sci _arttext\&pid=S2145-

94442014000200004\&lng=es\&tlng=es.
Torres O., J. A., y Duarte, J. E. (2016). Los procesos pedagógicos administrativos y los aspectos socio-culturales de inclusión y tecnopedagogía a través de las tendencias pedagógicas en educación a distancia y virtual. Revista de Investigación, Desarrollo e Innovación, Vol. 6, No. 2, 179-190. Doi: $10.19053 / 20278306.4606$

Valdés N., J. (2011). Lúdica y matemáticas a través de TICs para la práctica de operaciones con números enteros. Revista de Investigación, Desarrollo e Innovación RIDI, 1 (2), 17-27. Recuperado de: http://revistas.uptc.edu.co/index.php/investiga cion_duitama/article/view/1299

Valencia, N., Huertas, A., y Baracaldo, P. (2014). Los ambientes virtuales de aprendizaje: una revisión de publicaciones entre 2003 y 2013, desde la perspectiva de la pedagogía basada en la evidencia. Revista Colombiana de Educación, 73 - 103. 